Pacific

Journal of

Mathematics

ON ISOMETRIC AND CONFORMAL RIGIDITY OF SUBMANIFOLDS

SÉrgio L. Silva 


\title{
ON ISOMETRIC AND CONFORMAL RIGIDITY OF SUBMANIFOLDS
}

\author{
SÉRgio L. Silva
}

Dedicated to my mother for her 78th birthday

\begin{abstract}
In this article we introduce a new conformal invariant and we prove a conformal rigidity theorem which has no restriction on the size of the codimension. We also prove an isometric rigidity theorem whose assumptions are less restrictive than in Allendoerfer's theorem.
\end{abstract}

\section{Introduction.}

Let $f, g: M^{n} \rightarrow \mathbb{R}^{n+d}$ be two immersions of an $n$-dimensional differentiable manifold into Euclidean space. That $g$ is conformal (isometric) to $f$ means that the metrics induced on $M^{n}$ by $f$ and $g$ are conformal (isometric). We say that $f$ is conformally (isometrically) rigid if given any other conformal (isometric) immersion $g$ there exists a conformal (isometric) diffeomorphism $\Upsilon$ from an open subset of $\mathbb{R}^{n+d}$ to an open subset of $\mathbb{R}^{n+d}$ such that $g=\Upsilon \circ f$. In this case, we say that $f$ and $g$ are conformally (isometrically) congruent. It is then an interesting problem to determine conditions on $f$ which imply conformal (isometric) rigidity.

E. Cartan ([Ca1] , see also $[\mathbf{D a}])$ showed that when $n \geq 5$ a hypersurface $f: \quad M^{n} \rightarrow \mathbb{R}^{n+1}$ is "generically" conformally rigid. To be more specific, he proved that $f$ is conformally rigid when the maximal dimension of an umbilical subspace is at most $n-3$ at any point. Later, do Carmo and Dajczer ([C-D]) introduced a conformal invariant for immersions of arbitrary codimension, namely, the conformal s-nullity $\nu_{s}^{c}$, and generalized Cartan's result. More precisely, they showed that conformal rigidity holds whenever $d \leq 4, n \geq 2 d+3$ and $\nu_{s}^{c} \leq n-2 s-1$ for $1 \leq s \leq d$. As far as we know, it is still an open problem whether this result remains true for any codimension $d$. In this paper, we introduce a new conformal invariant, namely, the conformal type number $\tau_{f}^{c}$, and prove the following result which has no restriction on the size of the codimension.

Theorem 1.1. Let $f: M^{n} \rightarrow \mathbb{R}^{n+d}$ be an immersion. Assume that everywhere $\tau_{f}^{c}(p) \geq 3$ and that $\nu_{s}^{c}(p) \leq n-2 s-1$ for $1 \leq s \leq 3$. Suppose further that $n \geq 2 d+3$ if $d=1,2$. Then $f$ is conformally rigid. 
In relation to the above result see also Theorem 1.3 and Corollary 1.1.

Allendoerfer ([Al] $)$ showed that an isometric immersion with type number at least 3 everywhere is isometrically rigid. By using the notions of $k^{\text {th }}$ type number $\tau_{f}^{k}(p), 1 \leq k \leq d$, and $s$-nullity $\nu_{s}$, we obtain the following result whose assumptions are less restrictive (see Remark 2.1) than in Allendoerfer's theorem.

Theorem 1.2. Let $f: M^{n} \rightarrow \mathbb{R}^{n+d}, d \geq 2$, be an immersion. Assume that everywhere $\tau_{f}^{d-1}(p) \geq 3$ and $\nu_{s}(p) \leq n-2 s-1,1 \leq s \leq 3$, then $f$ is isometrically rigid.

This work is part of my Doctoral Thesis at IMPA. I would like to thank my research advisor, Professor Marcos Dajczer, for suggesting the problem and valuable remarks. I also would like to thank Professor Luis A. Florit and Professor Ruy Tojeiro for a number of helpful comments.

\section{Proof of Theorem 1.1.}

For a symmetric bilinear form $\beta: V \times V \rightarrow W$ we denote by $S(\beta)$ the subspace of $W$ given by

$$
S(\beta)=\operatorname{span}\{\beta(X, Y): X, Y \in V\},
$$

and by $N(\beta)$ the nullity space of $\beta$ defined as

$$
N(\beta)=\{n \in V: \beta(X, n)=0, \forall X \in V\} .
$$

Definition 1.1. Assume that $V$ and $W$ are endowed with positive definite inner products. We define the $k^{\text {th }}$ type number of $\beta, 1 \leq k \leq \operatorname{dim} W$, as being the largest integer $r$ for which there are $k$ vectors $\xi_{1}, \ldots, \xi_{k} \in W$ and $r$ vectors $X_{1}, \ldots, X_{r} \in V$ necessarily linearly independent such that the vectors $B_{\xi_{i}} X_{j}, 1 \leq i \leq k, 1 \leq j \leq r$, are linearly independent. Here $B_{\xi_{i}}: V \rightarrow V$ is given by $\left\langle B_{\xi_{i}} X, Y\right\rangle=\left\langle\beta(X, Y), \xi_{i}\right\rangle$. We point out that when $k=\operatorname{dim} W$ the $k^{\text {th }}$ type number does not depend on the basis of $W$.

Now let $f: M^{n} \rightarrow \tilde{M}^{n+d}$ be an immersion into a Riemannian manifold with vector valued second fundamental form $\alpha^{f}: T M \times T M \rightarrow T_{f}^{\perp} M$. The $k^{\text {th }}$ type number $\tau_{f}^{k}(p), 1 \leq k \leq d$, of $f$ at $p$ is defined as the $k^{\text {th }}$ type number of $\alpha^{f}$ at $p$. Observe that $\tau_{f}^{d}(p)$ is exactly the type number introduced by Allendoerfer.

Definition 1.2. We define the conformal type number $\tau_{f}^{c}(p)$ of $f$ at $p \in$ $M^{n}$ as being the integer

$$
\tau_{f}^{c}(p)=\max _{\eta \in T_{f(p)}^{\perp} M} \tau_{\eta}^{d}
$$

where $\tau_{\eta}^{d}$ denote the $d^{t h}$ type number of $\alpha^{f}-\langle,\rangle \eta$ at $p$. 
We claim that the conformal type number is a conformal invariant. In fact, let $\Upsilon$ be a conformal diffeomorphism of $\tilde{M}^{n+d}$ with conformal factor $\rho$, that is, $\left\langle\Upsilon_{*} X, \Upsilon_{*} Y\right\rangle=\rho^{2}\langle X, Y\rangle$. For $h=\Upsilon \circ f$, one easily verifies that

$$
\alpha^{h}=\Upsilon_{*} \alpha^{f}-\frac{1}{2(\rho \circ f)^{2}}\langle,\rangle \Upsilon_{*}\left(\nabla \rho^{2}\right)_{f}^{\perp},
$$

where $\nabla$ is the gradient operator. Thus the claim follows.

Given an $s$-dimensional subspace $U^{s} \subseteq T_{f(p)}^{\perp} M, 1 \leq s \leq d$, consider the bilinear form

$$
\alpha_{U^{s}}^{f}: T_{p} M \times T_{p} M \rightarrow U^{s}
$$

defined as $\alpha_{U^{s}}^{f}=P \circ \alpha^{f}$, where $P$ denotes the orthogonal projection of $T_{f(p)}^{\perp} M$ onto $U^{s}$. Endow $M^{n}$ with the induced metric. The conformal $s$-nullity $\nu_{s}^{c}(p)$ of $f$ at $p$ (see $[\mathbf{C}-\mathbf{D}]$ ) is the integer

$$
\nu_{s}^{c}(p)=\max _{U^{s} \subseteq T_{f(p)}^{\perp} M, \eta \in U^{s}}\left\{\operatorname{dim} N\left(\alpha_{U^{s}}^{f}-\langle,\rangle \eta\right)\right\} .
$$

The following result relate conformal type number and conformal $s$-nullity.

Proposition 1.1. Let $f: M^{n} \rightarrow \tilde{M}^{n+d}$ be an isometric immersion. If $\tau_{f}^{c}(p) \geq r$, then $\nu_{s}^{c}(p) \leq n-(s-1) r$ for $1 \leq s \leq d$.

Proof. Suppose $r \geq 1$ and $s \geq 2$. In any other case the result is immediate. Since $\tau_{f}^{c}(p) \geq r$, there exists $\eta \in T_{f(p)}^{\perp} M$ such that $\alpha^{f}-\langle,\rangle \eta$ has $d^{\text {th }}$ type number at least $r$. Consequently, for all basis $\xi_{1}, \ldots, \xi_{d}$ of $T_{f(p)}^{\perp} M$ there exist $r$ vectors $X_{1}, \ldots, X_{r}$ tangent at $p$ such that the vectors

$$
\left(A_{\xi_{i}}-\left\langle\eta, \xi_{i}\right\rangle I\right) X_{j}, \quad 1 \leq i \leq d, \quad 1 \leq j \leq r,
$$

are linearly independent. Let $U^{s} \subseteq T_{f(p)}^{\perp} M, 2 \leq s \leq d$, be an $s$-dimensional subspace and $\xi \in U^{s}$ an arbitrary vector. For the subspace $W=U^{s} \cap$ $(\operatorname{span}\{\eta-\xi\})^{\perp}$ it holds that $\operatorname{dim} W \geq s-1$. Take a basis $\xi_{1}, \ldots, \xi_{d}$ of $T_{f(p)}^{\perp} M$ such that $\xi_{1}, \ldots, \xi_{s}$ span $U^{s}$ and $\xi_{1}, \ldots, \xi_{s-1}$ are in $W$. Let $L$ be the subspace of $T_{p} M$ with dimension $(s-1) r$ given by

$$
L=\operatorname{span}\left\{\left(A_{\xi_{i}}-\left\langle\eta, \xi_{i}\right\rangle I\right) X_{j}, 1 \leq i \leq s-1,1 \leq j \leq r\right\} .
$$

For an arbitrary vector $v \in N\left(\alpha_{U^{s}}^{f}-\langle,\rangle \xi\right)$ and $1 \leq i \leq s$, we obtain that

$$
\begin{aligned}
0 & =\left\langle\alpha^{f}\left(v, X_{j}\right)-\left\langle v, X_{j}\right\rangle \xi, \xi_{i}\right\rangle \\
& =\left\langle\alpha^{f}\left(v, X_{j}\right)-\left\langle v, X_{j}\right\rangle \eta+\left\langle v, X_{j}\right\rangle(\eta-\xi), \xi_{i}\right\rangle .
\end{aligned}
$$

Thus, we have that $\left\langle\left(A_{\xi_{i}}-\left\langle\eta, \xi_{i}\right\rangle I\right) X_{j}, v\right\rangle=0,1 \leq i \leq s-1,1 \leq j \leq r$, that is, $N\left(\alpha_{U^{s}}^{f}-\langle,\rangle \xi\right) \subseteq L^{\perp}$. Since $U^{s} \subseteq T_{f(p)}^{\perp} M$ is arbitrary, the proof follows. 
Before proving Theorem 1.1 we recall some basic facts; from [Da] and $[\mathbf{D}-\mathbf{T}]$. Consider the Lorentz space $\mathbb{L}^{n+d+2}$, that is, Euclidean space $\mathbb{R}^{n+d+2}$ endowed with the metric $\langle$,$\rangle defined by$

$$
\langle X, X\rangle=-x_{1}^{2}+x_{2}^{2}+\cdots+x_{n+d+2}^{2}
$$

for $X=\left(x_{1}, x_{2}, \ldots, x_{n+d+2}\right)$. The light cone is the degenerate totally umbilical hypersurface of $\mathbb{L}^{n+d+2}$ defined by

$$
\mathbb{V}^{n+d+1}=\left\{X \in \mathbb{L}^{n+d+2}:\langle X, X\rangle=0, X \neq 0\right\} .
$$

Given $\zeta \in \mathbb{V}^{n+d+1}$ consider the hyperplane

$$
H_{\zeta}=\left\{X \in \mathbb{L}^{n+d+2}:\langle X, \zeta\rangle=1\right\}
$$

and the $(n+d)$-dimensional submanifold $H_{\zeta} \cap \mathbb{V}^{n+d+1}$. It is easy to see that the normal space to $H_{\zeta} \cap \mathbb{V}^{n+d+1}$ in $\mathbb{L}^{n+d+2}$ at $p$ is the Lorentzian plane $\mathbb{L}^{2}$ generated by $p$ and $\zeta$. Therefore, the metric induced by $\mathbb{L}^{n+d+2}$ on $H_{\zeta} \cap$ $\mathbb{V}^{n+d+1}$ is riemannian. The second fundamental form of this intersection is given by

$$
\alpha=-\langle,\rangle \zeta
$$

Using the Gauss equation, it follows that $H_{\zeta} \cap \mathbb{V}^{n+d+1}$ is an embedded flat riemannian submanifold of $\mathbb{L}^{n+d+2}$. Indeed, it can be checked that it is the image of an isometric embedding $J_{\zeta}: \mathbb{R}^{n+d} \rightarrow \mathbb{V}^{n+d+1}$.

The light cone is a very useful tool in the study of conformal immersions. Given any conformal immersion $g: M^{n} \rightarrow \mathbb{R}^{n+d}$ such that $\left\langle g_{*} X, g_{*} Y\right\rangle=$ $\phi_{g}^{2}\langle X, Y\rangle$, where $\phi_{g}>0$ is the conformal factor of $g$, we associate to $g$ an isometric immersion $G: M^{n} \rightarrow \mathbb{V}^{n+d+1} \subset \mathbb{L}^{n+d+2}$ by setting

$$
G=\frac{1}{\phi_{g}} J_{\zeta} \circ g
$$

for an arbitrary $\zeta \in \mathbb{V}^{n+d+1}$.

Conversely, any isometric immersion $G: M^{n} \rightarrow \mathbb{V}^{n+d+1}$ arises this way. In fact, choose $\zeta \in \mathbb{V}^{n+d+1}$ such that $\langle G, \zeta\rangle>0$. Define $g: M^{n} \rightarrow \mathbb{R}^{n+d}$ by setting

$$
J_{\zeta} \circ g=\frac{G}{\langle G, \zeta\rangle} .
$$

It is not difficult to verify that $g$ is a conformal immersion with conformal factor given by $1 /\langle G, \zeta\rangle$.

Now, let $g, f: M^{n} \rightarrow \mathbb{R}^{n+d}$ be conformal immersions and like previously discussed consider isometric immersions $G, F: M^{n} \rightarrow \mathbb{V}^{n+d+1}$ associated to them. If there exists an isometry $T: \mathbb{V}^{n+d+1} \rightarrow \mathbb{V}^{n+d+1}$ such that $F=T \circ G$, then $T$ induces a conformal diffeomorphism $\Upsilon$ from an open subset of $\mathbb{R}^{n+d}$ to an open subset of $\mathbb{R}^{n+d}$ defined by

$$
J_{\zeta} \circ \Upsilon=\frac{T \circ J_{\zeta}}{\left\langle T \circ J_{\zeta}, \zeta\right\rangle}
$$


which satisfies $f=\Upsilon \circ g$. In order to obtain such $T$ it suffices to construct a vector bundle isomorphism $\hat{T}: T_{G}^{\perp} M \rightarrow T_{F}^{\perp} M$ preserving metrics, second fundamental forms and normal connections. Here, $T_{G}^{\perp} M$ and $T_{F}^{\perp} M$ stand for the normal bundles of $G$ and $F$, respectively, in $\mathbb{L}^{n+d+2}$. From the fundamental theorem for isometric immersions adapted to the Lorentzian case we conclude that there exists an isometry $\bar{T}: \mathbb{L}^{n+d+2} \rightarrow \mathbb{L}^{n+d+2}$ such that $F=\bar{T} \circ G$. Then we take $T$ as the restriction of $\bar{T}$ to $\mathbb{V}^{n+d+1}$.

Proof of Theorem 1.1. We only have to deal with the case of codimension $d \geq$ 5. If $\tau_{f}^{c}(p) \geq 3$ then $n \geq 3 d$ and can be easily deduced from Proposition 1.1 that $\nu_{s}^{c}(p) \leq n-2 s-1$ when $s \geq 4$. Consequently, under our assumptions we always have that $n \geq 2 d+3$ and $\nu_{s}^{c}(p) \leq n-2 s-1$. Thus, the following result already reported in the introduction applies for $d \leq 4$.

Theorem $1.3([\mathbf{C}-\mathbf{D}])$. Let $f: M^{n} \rightarrow \mathbb{R}^{n+d}$ be an immersion where $d \leq 4$ and $n \geq 2 d+3$. Assume that $\nu_{s}^{c}(p) \leq n-2 s-1$ for all $p \in M^{n}$ and every integer $s, 1 \leq s \leq d$. Then $f$ is conformally rigid.

Let $g: M^{n} \rightarrow \mathbb{R}^{n+d}$ be any immersion conformal to $f$ and $G: M^{n} \rightarrow$ $\mathbb{V}^{n+d+1}$ its associated isometric immersion. We may assume that $M^{n}$ is endowed with the metric induced by $f$. Taking the derivative of $\langle G, G\rangle=0$, we see that the null vector field $G$ is normal to the immersion $G$. The normal field $G$ also satisfies $A_{G}^{G}=-I$. The normal bundle of $G$ is given by the orthogonal direct sum

$$
T_{G}^{\perp} M=T_{g}^{\perp} M \oplus \mathbb{L}^{2}
$$

where $T_{g}^{\perp} M$ is identified with $\left(J_{\zeta}\right)_{*} T_{g}^{\perp} M$ and $\mathbb{L}^{2}$ is a Lorentzian plane bundle which contains $G$. We can easily see that there exists a unique orthogonal frame $\{\xi, \eta\}$ of $\mathbb{L}^{2}$ with $|\xi|^{2}=-1$ such that

$$
G=\xi+\eta \text {. }
$$

Writing $\alpha^{G}$ in terms of this orthogonal frame we obtain

$$
\alpha^{G}=-\left\langle\alpha^{G}, \xi\right\rangle \xi+\left\langle\alpha^{G}, \eta\right\rangle \eta+\left(\alpha^{G}\right)^{*}
$$

where $\left(\alpha^{G}\right)^{*}=\left(1 / \phi_{g}\right)\left(J_{\zeta}\right)_{*} \alpha^{g}$ is the $T_{g}^{\perp} M$-component of $\alpha^{G}$.

Given an $m$-dimensional real vector space $W$ endowed with a non-degenerate inner product $\langle$,$\rangle of index r$, that is, the maximal dimension of a subspace of $W$ where $\langle$,$\rangle is negative definite, we say that W$ is of type $(r, q)$ and we write $W^{(r, q)}$ with $q=m-r$.

At $p \in M^{n}$, let

$$
W=T_{f(p)}^{\perp} M \oplus \operatorname{span}\{\xi(p)\} \oplus \operatorname{span}\{\eta(p)\} \oplus T_{g(p)}^{\perp} M
$$

be endowed with the natural metric of type $(d+1, d+1)$ which is negative definite on $T_{f(p)}^{\perp} M \oplus \operatorname{span}\{\xi(p)\}$. We also define a symmetric bilinear form 
$\beta: T M \times T M \rightarrow W$ setting $\beta=\alpha^{f}+\alpha^{G}$, i.e.,

$$
\beta=\alpha^{f}-\left\langle\alpha^{G}, \xi\right\rangle \xi+\left\langle\alpha^{G}, \eta\right\rangle \eta+\left(\alpha^{G}\right)^{*} .
$$

The Gauss equations for $f$ and $G$ imply that $\beta$ is flat, i.e.,

$$
\langle\beta(X, Y), \beta(Z, U)\rangle=\langle\beta(X, U), \beta(Y, Z)\rangle, \quad \forall X, Y, Z, U \in T M .
$$

Observe also that $\beta(X, X) \neq 0$ for all $X \neq 0$, because $A_{\xi+\eta}^{G}=-I$.

Lemma 1.1. The bilinear form $\beta$ is null, that is,

$$
\langle\beta(X, Y), \beta(Z, U)\rangle=0, \quad \forall X, Y, Z, U \in T M .
$$

Proof. Fixed $p \in M^{n}$, set $V:=T_{p} M$ and for each $X \in V$ define the linear map

$$
\beta(X): V \rightarrow W
$$

by setting $\beta(X)(v)=\beta(X, v)$ for all $v \in V$. For simplicity of notation, we omit the $p$. The kernel and image of $\beta(X)$ are denoted by $\operatorname{ker} \beta(X)$ and $\beta(X, V)$, respectively. We say that $X$ is a regular element of $\beta$ if

$$
\operatorname{dim} \beta(X, V)=\max _{Z \in V} \operatorname{dim} \beta(Z, V) .
$$

The set of regular elements of $\beta$ is denoted by $R E(\beta)$. For each $X \in V$, set $U(X)=\beta(X, V) \cap \beta(X, V)^{\perp}$ and define

$$
R E^{*}(\beta)=\left\{Y \in R E(\beta): \operatorname{dim} U(Y)=d_{0}\right\}
$$

where $d_{0}=\min \{\operatorname{dim} U(Y): Y \in R E(\beta)\}$.

We will need the following from $[\mathbf{D a}]$.

Sublemma 1.1. The set $R E^{*}(\beta)$ is open and dense in $V$ and

$$
\beta(\operatorname{ker} \beta(X), V) \subseteq U(X), \quad \forall X \in R E(\beta) .
$$

Now recall that a vector subspace $L$ of $W$ is said to be degenerate when satisfies $L \cap L^{\perp} \neq\{0\}$ and isotropic if $\langle L, L\rangle=0$. We also have that

$$
\operatorname{dim} L+\operatorname{dim} L^{\perp}=\operatorname{dim} W \quad \text { and } \quad L^{\perp \perp}=L .
$$

It follows easily from (1.1), $\operatorname{dim} W=2 d+2$ and the definition of $U(X)$ that $d_{0} \leq d+1$. We separate the proof in two cases, namely, $d_{0}=d+1$ and $d_{0} \leq d$.

Case I. $d_{0}=d+1$. In this case, $\operatorname{dim} U(X)=d+1$ for all $X \in R E^{*}(\beta)$. Then, $U(X)=\beta(X, V)=\beta(X, V)^{\perp}$ due to (1.1). Using the density of $R E^{*}(\beta)$, we get

$$
\langle\beta(X, Y), \beta(X, Z)\rangle=0, \quad \forall X, Y, Z \in V,
$$

and the bilinearity of $\beta$ yields the claim.

Case II. $d_{0} \leq d$. To deal with this case we need several facts.

Assertion 1. $\operatorname{dim} S(\beta) \cap S(\beta)^{\perp} \geq d-2$. 
Since $\tau_{f}^{c}(p) \geq 3$, there exists $\eta \in T_{f(p)}^{\perp} M$ such that $\alpha^{f}-\langle,\rangle \eta$ has type number at least 3 . Fix a basis $\xi_{1}, \ldots, \xi_{d}$ of $T_{f(p)}^{\perp} M$ and vectors $X_{1}, X_{2}, X_{3} \in$ $V$ so that the $A_{\xi_{i}}^{\lambda_{i}, f} X_{j}=\left(A_{\xi_{i}}^{f}+\lambda_{i} I\right) X_{j}, \lambda_{i}=\left\langle\eta, \xi_{i}\right\rangle, 1 \leq i \leq d, 1 \leq j \leq 3$, are linearly independent. Define

$$
\tilde{L}=\left(\operatorname{span}\left\{A_{\xi_{i}}^{\lambda_{i}, f} X_{j}: 1 \leq i \leq d, 1 \leq j \leq 3\right\}\right)^{\perp} .
$$

We have that $\operatorname{dim} \tilde{L}=n-3 d$ and that $Z \in \tilde{L}$ if and only if

$$
\left\langle\alpha^{f}\left(X_{j}, Z\right), \xi_{i}\right\rangle+\lambda_{i}\left\langle X_{j}, Z\right\rangle=0, \quad \forall i, j .
$$

By definition of $\beta$ we have for $Z \in \operatorname{ker} \beta\left(X_{j}\right)$ that

$$
\alpha^{f}\left(X_{j}, Z\right)=0 \text { and }\left\langle\alpha^{G}\left(X_{j}, Z\right), \xi\right\rangle=\left\langle\alpha^{G}\left(X_{j}, Z\right), \eta\right\rangle=0 .
$$

Since $A_{\eta+\xi}^{G}=-I$, we get $\left\langle X_{j}, Z\right\rangle=-\left\langle\alpha^{G}\left(X_{j}, Z\right), \eta+\xi\right\rangle=0$. Hence,

$$
\bigcap_{h=1}^{3} \operatorname{ker} \beta\left(X_{h}\right) \subseteq \tilde{L} .
$$

We can assume that $X_{1}, X_{2}, X_{3} \in R E^{*}(\beta)$ by Sublemma 1.1. Unless otherwise stated, from now on the indexes $i, j, k \in\{1,2,3\}$ are all distinct. Moreover, for simplicity of notation we denote the map $\beta\left(X_{i}\right)$ and its image $\beta\left(X_{i}, V\right)$ by $\beta_{i}$ and $\operatorname{Im} \beta_{i}$, respectively. Take the maps

$$
\Gamma_{k}: \operatorname{ker} \beta_{i} \cap \operatorname{ker} \beta_{j} \rightarrow U\left(X_{i}\right) \cap U\left(X_{j}\right)
$$

as being the restriction of $\beta_{k}$ to $\operatorname{ker} \beta_{i} \cap \operatorname{ker} \beta_{j}$ and

$$
\Gamma_{i j}: \operatorname{ker} \beta_{j} \rightarrow U\left(X_{j}\right)
$$

as the restriction of $\beta_{i}$ to $\operatorname{ker} \beta_{j}$. By Sublemma 1.1 the maps $\Gamma_{k}$ and $\Gamma_{i j}$ are well defined. Setting $U_{j}=U\left(X_{j}\right)$ and $U_{i j}=U_{i} \cap U_{j}$, we have that

(1.3) $\operatorname{Im} \Gamma_{k} \subseteq \operatorname{Im} \Gamma_{k j} \subseteq U_{j}$ and $\operatorname{Im} \Gamma_{k} \subseteq U_{i j} \subseteq U_{j}, \quad \forall i, j, k \in\{1,2,3\}$.

Define

$$
\rho=\operatorname{dim}\left(\operatorname{Im} \beta_{i}\right)^{\perp}-d_{0} \quad \text { and } \quad \theta_{i}^{j}=d_{0}-\operatorname{dim} \operatorname{Im} \Gamma_{i j} .
$$

A simple calculation shows that

$$
\operatorname{dim} \operatorname{ker} \Gamma_{i j}=n-2 d-2+\rho+\theta_{i}^{j} .
$$

Setting

$$
\gamma_{k}=d_{0}-\operatorname{dim} \operatorname{Im} \Gamma_{k}
$$

and using that $\operatorname{ker} \Gamma_{i j}=\operatorname{ker} \beta_{i} \cap \operatorname{ker} \beta_{j}$, we obtain that

$$
\operatorname{dim} \operatorname{ker} \Gamma_{k}=n-2 d-d_{0}-2+\rho+\theta_{i}^{j}+\gamma_{k} .
$$


Since

$$
\operatorname{ker} \Gamma_{k}=\bigcap_{h=1}^{3} \operatorname{ker} \beta_{h},
$$

the last equality shows that the sums $\theta_{i}^{j}+\gamma_{k}$ are independent of the indexes. This and (1.2) imply that $n-3 d \geq n-2 d-d_{0}-2+\rho+\theta_{i}^{j}+\gamma_{k}$. Hence

$$
d-d_{0}-2+\left(\rho+\theta_{i}^{j}+\gamma_{k}\right) \leq 0 .
$$

Since the integers $\rho, \theta_{i}^{j}$ and $\gamma_{k}$ are nonnegative, it follows that $d_{0} \geq d-2$. We have to analyze three possibilities for $d_{0}$.

II.(a). $d_{0}=d-2$. In this case $\rho=\theta_{i}^{j}=\gamma_{k}=0$ by (1.7). Observe that $\gamma_{k}=0$ and (1.3) yield that

$$
\operatorname{Im} \Gamma_{k}=U_{j}=U_{i} .
$$

We show that $\operatorname{Im} \Gamma_{i} \subseteq S(\beta) \cap S(\beta)^{\perp}$. An arbitrary element in $\operatorname{Im} \Gamma_{i}$ is given by $\beta\left(X_{i}, X_{0}\right)$ with $X_{0} \in \operatorname{ker} \Gamma_{j k}$. Since $\beta\left(X_{0}, Y\right) \in U_{j k}$ by Sublemma 1.1, we get using (1.8) that

$$
\left\langle\beta\left(X_{i}, X_{0}\right), \beta(Y, Z)\right\rangle=\left\langle\beta\left(X_{i}, Z\right), \beta\left(X_{0}, Y\right)\right\rangle=0, \quad \forall Y, Z \in V .
$$

We conclude that $\operatorname{dim} S(\beta) \cap S(\beta)^{\perp} \geq d_{0}=d-2$.

II.(b). $d_{0}=d-1$. In this case $\rho+\theta_{i}^{j}+\gamma_{k} \leq 1$. We have to consider two sub-cases.

(b).1. There exist indexes such that $U_{i j}=U_{k j}$. Like in II.(a) we conclude that $\operatorname{Im} \Gamma_{i} \subseteq S(\beta) \cap S(\beta)^{\perp}$ and $\operatorname{dim} S(\beta) \cap S(\beta)^{\perp} \geq d-2$.

(b).2. Suppose that $U_{i j} \neq U_{k j}$ for all $i, j, k$. This implies that $U_{i} \neq U_{j}$ for all $i, j$. It follows from (1.3) that $\gamma_{k}=1$. In particular,

$$
\operatorname{dim} \operatorname{Im} \Gamma_{k}=d-2 \text { and } \operatorname{Im} \Gamma_{k}=U_{i j} .
$$

Being $\theta_{i}^{j}=0$, then (1.3) gives

$$
U_{j}=\operatorname{Im} \Gamma_{i j} \subset \operatorname{Im} \beta_{i} .
$$

Due to $\rho=0$ and $\operatorname{Im} \beta_{i} \subseteq U_{i}^{\perp}$, we deduce that

$$
\operatorname{Im} \beta_{i}=U_{i}^{\perp} \text {. }
$$

The assumption in (b).2 jointly to (1.9) and (1.11) imply that

$$
U_{k i} \not \subset U_{k j} \text { and } \operatorname{Im} \beta_{i} \not \subset \operatorname{Im} \beta_{j} \text {. }
$$

It is not difficult to see that $U_{j}=U_{i j}+U_{k j} \subseteq U_{i}+U_{k}$ due to the assumption in (b).2, (1.9) and $\operatorname{dim} U_{j}=d-1$. For all $i$ and $j$, the subspace $U_{i}+U_{j}$ has dimension $d$ by the formula

$$
\operatorname{dim}\left(L_{1}+L_{2}\right)=\operatorname{dim} L_{1}+\operatorname{dim} L_{2}-\operatorname{dim} L_{1} \cap L_{2} .
$$


Therefore,

$$
U_{i}+U_{j}=U_{i}+U_{k}
$$

and, consequently,

$$
\operatorname{Im} \beta_{i} \cap \operatorname{Im} \beta_{j}=\operatorname{Im} \beta_{i} \cap \operatorname{Im} \beta_{k}
$$

by (1.11) and the formula

$$
\left(\sum_{h} L_{h}\right)^{\perp}=\bigcap_{h} L_{h}^{\perp},
$$

valid for any arbitrary finite number of subspaces. If $v \in V$ and $w \in \operatorname{ker} \beta_{j}$, then

$$
\left\langle\beta\left(X_{k}, v\right), \beta\left(X_{i}, w\right)\right\rangle=\left\langle\beta\left(X_{k}, X_{i}\right), \beta(v, w)\right\rangle=0
$$

since $\beta\left(X_{k}, X_{i}\right) \in \operatorname{Im} \beta_{j}$ by (1.15) and $\beta(v, w) \in U_{j}$ by Sublemma 1.1. The last equality, (1.10) and (1.11) imply that $\operatorname{Im} \beta_{k} \subset\left(\operatorname{Im} \Gamma_{i j}\right)^{\perp}=U_{j}^{\perp}=\operatorname{Im} \beta_{j}$ which is in contradiction to (1.12).

II.(c). $d_{0}=d$. It follows from (1.7) that $\rho+\theta_{i}^{j}+\gamma_{k} \leq 2$. Like in II.(b), we consider two sub-cases.

(c).1. There exist indexes such that $U_{i j}=U_{k j}$. Proceeding analogous to II.(a) gives $\operatorname{dim} S(\beta) \cap S(\beta)^{\perp} \geq d-2$.

(c).2. Suppose that $U_{i j} \neq U_{k j}$ for all $i, j, k$. This implies that $U_{i} \neq U_{j}$ for all $i, j$. It follows from (1.3) that $1 \leq \gamma_{k} \leq 2$, then $\rho+\theta_{i}^{j} \leq 1$. From (1.6), we conclude that $d-2 \leq \operatorname{dim} \operatorname{Im} \Gamma_{k} \leq \operatorname{dim} U_{i j} \leq d-1$. If there exist indexes $i$ and $j$ such that $\operatorname{dim} U_{i j}=d-2$, then $\gamma_{k}=2, \rho=0$ and $\theta_{i}^{j}=\theta_{j}^{i}=0$. Thus, $U_{j}=\operatorname{Im} \Gamma_{i j} \subset \operatorname{Im} \beta_{i}$ and $U_{i}=\operatorname{Im} \Gamma_{j i} \subset \operatorname{Im} \beta_{j}$ by (1.3). We have that $\operatorname{dim}\left(U_{i}+U_{j}\right)=d+2$ by (1.13). Here the subspace $U_{i}+U_{j}$ is isotropic since $U_{i}$ and $U_{j}$ are isotropic and $U_{j}$, being a subset of $\operatorname{Im} \beta_{i}$, is orthogonal to $U_{i}$. But this is not possible due to (1.1). Then we can assume $\operatorname{dim} U_{i j}=d-1$ for all $i$ and $j$. In this case, $\operatorname{dim}\left(U_{i}+U_{j}\right)=d+1$ and (1.14) holds.

(c).2.1. First suppose there exists $k$ with $\gamma_{k}=2$. Thus, $\theta_{i}^{j}=\rho=0$. Being $\rho=0,(1.11)$ holds. From (1.14) and (1.16), we have that (1.15) also holds. Like in (b).2, we obtain a contradiction.

(c).2.2. Consider $\gamma_{k}=1$ for all $k$. In this case the $\theta_{i}^{j}$,s are independent from the indexes. There are three possibilities:

(c).2.2.1. Suppose that $\rho=\theta_{i}^{j}=0$. Similar to (b).2 we have a contradiction.

(c).2.2.2. Suppose that $\rho=0$ and $\theta_{i}^{j}=1$. Being $\rho=0$, then (1.11), (1.12) and (1.15) hold. Further,

$$
\operatorname{dim} \operatorname{ker} \Gamma_{i j}=n-2 d-1
$$


by (1.5) and

$$
\operatorname{dim} \operatorname{Im} \beta_{i}=d+2, \quad \text { hence } \operatorname{dim} \operatorname{ker} \beta_{i}=n-d-2 .
$$

It holds that $\operatorname{Im} \Gamma_{k j}=\operatorname{Im} \Gamma_{k}=U_{i j}$ from the assumption in (c).2, $\gamma_{k}=$ $\theta_{k}^{j}=1$ and (1.3). We claim that $U_{i} \subset \operatorname{Im} \beta_{j}$. Otherwise, we have that $U_{i j}=U_{i} \cap \operatorname{Im} \beta_{j}$ since $d-1=\operatorname{dim} U_{i j} \leq \operatorname{dim}\left(U_{i} \cap \operatorname{Im} \beta_{j}\right) \leq d-1$. Using (1.15), we conclude that

$U_{i j}=U_{i} \cap \operatorname{Im} \beta_{j}=U_{i} \cap \operatorname{Im} \beta_{i} \cap \operatorname{Im} \beta_{j}=U_{i} \cap \operatorname{Im} \beta_{i} \cap \operatorname{Im} \beta_{k}=U_{i} \cap \operatorname{Im} \beta_{k}=U_{i k}$ contradicting (c).2, and the claim follows. From (1.13), (1.17) and (1.18), we deduce that

$$
\operatorname{dim}\left(\operatorname{ker} \beta_{i}+\operatorname{ker} \beta_{j}\right)=n-3 .
$$

The vector $X_{i}$ satisfies that $X_{i} \notin\left(\operatorname{ker} \beta_{i}+\operatorname{ker} \beta_{j}\right)$. Otherwise, using Sublemma 1.1, we obtain the following contradiction due to dimensions:

$$
\operatorname{Im} \beta_{i} \subset \beta\left(\operatorname{ker} \beta_{i}+\operatorname{ker} \beta_{j}, V\right) \subseteq U_{i}+U_{j} .
$$

The vector $X_{j} \notin \operatorname{span}\left\{X_{i}\right\} \oplus\left(\operatorname{ker} \beta_{i}+\operatorname{ker} \beta_{j}\right)$. Otherwise,

$$
\operatorname{Im} \beta_{j} \subset \beta\left(\operatorname{span}\left\{X_{i}\right\} \oplus\left(\operatorname{ker} \beta_{i}+\operatorname{ker} \beta_{j}\right), V\right) \subseteq \operatorname{Im} \beta_{i}+U_{i}+U_{j}=\operatorname{Im} \beta_{i}
$$

which is in contradiction with (1.12). It hold that

$$
\operatorname{Im} \beta_{i}+\operatorname{Im} \beta_{j}=U_{i j}^{\perp} \quad \text { and } \quad \operatorname{Im} \beta_{i} \cap \operatorname{Im} \beta_{j}=\left(U_{i}+U_{j}\right)^{\perp}
$$

by (1.11) and (1.16). We assert that

$$
\operatorname{Im} \beta_{k} \not \subset \operatorname{Im} \beta_{i}+\operatorname{Im} \beta_{j} .
$$

On the contrary, $\operatorname{Im} \beta_{k}+\operatorname{Im} \beta_{j} \subseteq \operatorname{Im} \beta_{i}+\operatorname{Im} \beta_{j}$ and, since both spaces have dimension $d+3$, then the equality holds. But this jointly to (1.20) contradicts the hypothesis in (c).2. Also $X_{k} \notin \operatorname{span}\left\{X_{i}, X_{j}\right\} \oplus\left(\operatorname{ker} \beta_{i}+\operatorname{ker} \beta_{j}\right)$ due to (1.21). It follows from (1.19) that

$$
V=\operatorname{span}\left\{X_{1}, X_{2}, X_{3}\right\} \oplus\left(\operatorname{ker} \beta_{i}+\operatorname{ker} \beta_{j}\right) .
$$

The above equality gives that $S(\beta)=\sum_{h=1}^{3} \operatorname{Im} \beta_{h}$. This fact, together with (1.11), (1.16) and $\left(\bigcap_{h=1}^{3} U_{h}\right) \subseteq S(\beta)$ prove that $S(\beta) \cap S(\beta)^{\perp}=\left(\bigcap_{h=1}^{3} U_{h}\right)$. Therefore, $\operatorname{dim} S(\beta) \cap S(\beta)^{\perp}=\operatorname{dim}\left(\bigcap_{h=1}^{3} U_{h}\right)=d-2$.

(c).2.2.3. Finally, suppose that $\rho=1$ and $\theta_{i}^{j}=0$. Then $\operatorname{dim}\left(\operatorname{Im} \beta_{i}\right)^{\perp}=$ $d+1$ by (1.4). Thus, $\operatorname{dim} \operatorname{Im} \beta_{i}=d+1$ by (1.1). From (1.3), we have $U_{j}=\operatorname{Im} \Gamma_{i j} \subset \operatorname{Im} \beta_{i}$ since $\theta_{i}^{j}=0$. These facts and $U_{i} \neq U_{j}$ imply that $U_{i}+U_{j}=\operatorname{Im} \beta_{i}$. Arguing as in (c).2 we obtain that $U_{i}+U_{j}$ is isotropic. So $U_{i}=\operatorname{Im} \beta_{i} \cap\left(\operatorname{Im} \beta_{i}\right)^{\perp}=\operatorname{Im} \beta_{i}$, and we get a contradiction due to dimensions. 
Assertion 2. There exist an orthogonal decomposition

$$
W=W_{1}^{(\ell, \ell)} \oplus W_{2}^{(d-\ell+1, d-\ell+1)}, \quad \ell \geq d-2,
$$

and symmetric bilinear forms $\omega_{j}: V \times V \rightarrow W_{j}, 1 \leq j \leq 2$, satisfying

$$
\beta=\omega_{1} \oplus \omega_{2}
$$

such that:

i) $\omega_{1}$ is nonzero and null with respect to $\langle$,$\rangle and$

ii) $\omega_{2}$ is flat with $\operatorname{dim} N\left(\omega_{2}\right) \geq n-\operatorname{dim} W_{2}$.

Let $v_{1}, \ldots, v_{\ell}$ a basis of $S(\beta) \cap S(\beta)^{\perp}$. There exists (see [Da], p. 83) a pseudo-orthonormal basis $v_{1}, \ldots, v_{\ell}, \hat{v}_{1}, \ldots, \hat{v}_{\ell}, \theta_{1}, \ldots, \theta_{2(d-\ell+1)}$ of $W$ satisfying that $\left\langle v_{i}, v_{j}\right\rangle=\left\langle\hat{v}_{i}, \hat{v}_{j}\right\rangle=\left\langle\theta_{i}, v_{j}\right\rangle=\left\langle\theta_{i}, \hat{v}_{j}\right\rangle=0,\left\langle v_{i}, \hat{v}_{j}\right\rangle=\delta_{i j}$ and that $\left\langle\theta_{i}, \theta_{j}\right\rangle= \pm \delta_{i j}$. Defining

$$
W_{1}=\operatorname{span}\left\{v_{1}, \ldots, v_{\ell}, \hat{v}_{1}, \ldots, \hat{v}_{\ell}\right\}, \quad W_{2}=\operatorname{span}\left\{\theta_{1}, \ldots, \theta_{2(d-\ell+1)}\right\}
$$

and

$$
\beta=\sum_{i=1}^{\ell} \phi_{i} v_{i}+\sum_{i=1}^{\ell} \psi_{i} \hat{v}_{i}+\sum_{i=1}^{2(d-\ell+1)} \kappa_{i} \theta_{i}
$$

we have that $\psi_{i}=\left\langle\beta, v_{i}\right\rangle=0$. Set

$$
\omega_{1}=\sum_{i=1}^{\ell} \phi_{i} v_{i} \text { and } \omega_{2}=\sum_{i=1}^{2(d-\ell+1)} \kappa_{i} \theta_{i} .
$$

Since $\ell=\operatorname{dim} S(\beta) \cap S(\beta)^{\perp} \geq d-2 \geq 3$, then $\omega_{1}$ is nonzero. It is easy to verify that $\omega_{1}, \omega_{2}$ are symmetric bilinear forms such that $\omega_{1}$ is null and $\omega_{2}$ is flat. In order to see that $S\left(\omega_{2}\right)$ is non-degenerate, let $\sum_{i} \omega_{2}\left(X_{i}, Y_{i}\right) \in W_{2}$ be an arbitrary element in $S\left(\omega_{2}\right) \cap S\left(\omega_{2}\right)^{\perp}$. For all $v, w \in V$, we get

$$
\left\langle\sum_{i} \omega_{2}\left(X_{i}, Y_{i}\right), \beta(v, w)\right\rangle=\left\langle\sum_{i} \omega_{2}\left(X_{i}, Y_{i}\right), \omega_{2}(v, w)\right\rangle=0 .
$$

Therefore, $\sum_{i} \omega_{2}\left(X_{i}, Y_{i}\right) \in S(\beta) \cap S(\beta)^{\perp}$. Hence, $\sum_{i} \omega_{2}\left(X_{i}, Y_{i}\right) \in W_{1}$. Thus,

$$
\sum_{i} \omega_{2}\left(X_{i}, Y_{i}\right) \in W_{1} \cap W_{2}=\{0\} .
$$

Since the subspace $S\left(\omega_{2}\right)$ is non-degenerate and $d-\ell+1 \leq 3$, the inequality $\operatorname{dim} N\left(\omega_{2}\right) \geq n-\operatorname{dim} W_{2}$ is a consequence of the following result whose proof is part of the arguments for the Main Lemma 2.2 in ([C-D], pp. 968-974).

Sublemma 1.2. Let $\sigma: V_{1} \times V_{1} \rightarrow W^{(r, r)}$ be a nonzero flat symmetric bilinear form. Assume $r \leq 5$ and $\operatorname{dim} N(\sigma)<\operatorname{dim} V_{1}-2 r$. Then $S(\sigma)$ is degenerate.

Now Lemma 1.1 is a consequence of the following fact. 
Assertion 3. The bilinear form $\omega_{2}$ is zero.

Suppose on the contrary that $\ell \leq d$. Set $v_{i}=\gamma_{i}+b_{i} \xi+c_{i} \eta+\delta_{i}, 1 \leq i \leq \ell$, where $\gamma_{i} \in T_{f}^{\perp} M$ and $\delta_{i} \in T_{g}^{\perp} M$. Let $\hat{L}$ be the orthogonal complement in $T_{f(p)}^{\perp} M$ of the subspace $L=\operatorname{span}\left\{\gamma_{i}: 1 \leq i \leq \ell\right\}$. If the vectors $\gamma_{i}$ are linearly dependent, then $\operatorname{dim} \hat{L} \geq d-\ell+1 \geq 1$. For any $n \in N\left(\omega_{2}\right), v \in V$ and $u \in \hat{L}$, it holds that

$$
\left\langle\alpha^{f}(n, v), u\right\rangle=\langle\beta(n, v), u\rangle=\left\langle\omega_{1}(n, v), u\right\rangle=0 .
$$

Hence,

$$
\nu_{\operatorname{dim} \hat{L}}^{c} \geq \operatorname{dim} N\left(\alpha_{\hat{L}}^{f}\right) \geq \operatorname{dim} N\left(\omega_{2}\right) \geq n-2(d-\ell+1) \geq n-2 \operatorname{dim} \hat{L},
$$

which is in contradiction with the hypothesis on the conformal $(\operatorname{dim} \hat{L})$ nullity when $1 \leq \operatorname{dim} \hat{L} \leq 3$ and with the Proposition 1.1 when $\operatorname{dim} \hat{L} \geq 4$.

Now, since the vectors $\gamma_{i}$ are linearly independent, then $\operatorname{dim} \hat{L}=\bar{d}-\ell$. The definition of $\beta$ gives

$$
\left\langle\alpha^{G}(n, v), \eta\right\rangle=\langle\beta(n, v), \eta\rangle=\left\langle\omega_{1}(n, v), \eta\right\rangle=\sum_{i=1}^{\ell} c_{i} \phi_{i}
$$

and

$$
\left\langle\alpha^{G}(n, v), \xi\right\rangle=\langle\beta(n, v), \xi\rangle=\left\langle\omega_{1}(n, v), \xi\right\rangle=-\sum_{i=1}^{\ell} b_{i} \phi_{i} .
$$

Therefore,

$$
-\langle n, v\rangle=\left\langle n, A_{\eta+\xi}^{G} v\right\rangle=\sum_{i=1}^{\ell}\left(c_{i}-b_{i}\right) \phi_{i} .
$$

Fix $j \in\{1, \ldots, \ell\}$ and consider the hyperplane $L_{j} \subset L$ given by

$$
L_{j}=\operatorname{span}\left\{\gamma_{1}, \ldots, \gamma_{j-1}, \gamma_{j+1}, \ldots, \gamma_{\ell}\right\} .
$$

Let $\mu_{j}$ be the orthogonal projection of $\gamma_{j}$ onto the orthogonal complement of $L_{j}$ in $L$. Observing that $\left\langle\mu_{i}, \gamma_{j}\right\rangle=\delta_{i j}\left|\mu_{i}\right|^{2}$, it is not difficult to see that the vectors $\mu_{1}, \ldots, \mu_{\ell}$ are linearly independent. Let $\gamma \in L \subset T_{f(p)}^{\perp} M$ be defined as

$$
\gamma=\sum_{i=1}^{\ell} \frac{c_{i}-b_{i}}{\left|\mu_{i}\right|^{2}} \mu_{i}
$$

Then (1.22) yields that $\gamma \neq 0$. Set $U^{d-\ell+1}=\operatorname{span}\{\gamma\} \oplus \hat{L}$. It holds that

$$
\left\langle\alpha^{f}(n, v)+\frac{\gamma}{|\gamma|^{2}}\langle n, v\rangle, \gamma\right\rangle=0 \quad \text { and } \quad\left\langle\alpha^{f}(n, v)+\frac{\gamma}{|\gamma|^{2}}\langle n, v\rangle, u\right\rangle=0
$$


for all $n \in N\left(\omega_{2}\right), v \in V$ and $u \in \hat{L}$. Then,

$$
\nu_{d-\ell+1}^{c} \geq \operatorname{dim} N\left(\alpha_{U^{d-\ell+1}}^{f}+\frac{\gamma}{|\gamma|^{2}}\langle,\rangle\right) \geq \operatorname{dim} N\left(\omega_{2}\right) \geq n-2(d-\ell+1) .
$$

This is in contradiction with the hypothesis on the conformal $(d-\ell+1)$ nullity.

Assertion 4. There exists an orthonormal basis $\gamma_{1}, \ldots, \gamma_{d}$ of $T_{f(p)}^{\perp} M$ and a pseudo-orthonormal basis $G, \mu_{1}, \ldots, \mu_{d+1}$ of $T_{G(p)}^{\perp} M$, with $\left\langle G, \mu_{1}\right\rangle=$ $1,\left\langle\mu_{1}, \mu_{1}\right\rangle=0$, such that

$$
\alpha^{G}=-\langle,\rangle \mu_{1}+\sum_{j=1}^{d}\left\langle\alpha^{f}, \gamma_{j}\right\rangle \mu_{j+1} .
$$

Define

$$
\tilde{\beta}=\alpha^{f}-\left\langle\alpha^{G}, \xi\right\rangle \xi, \quad \bar{\beta}=\left\langle\alpha^{G}, \eta\right\rangle \eta+\left(\alpha^{G}\right)^{*} .
$$

Since $\beta$ is null, we conclude that

$$
\langle\tilde{\beta}(X, Y), \tilde{\beta}(Z, U)\rangle=\langle\bar{\beta}(X, Y), \bar{\beta}(Z, U)\rangle, \quad \forall X, Y, Z, U \in T_{p} M,
$$

where we have changed the sign of the metric in $T_{f(p)}^{\perp} M \oplus \operatorname{span}\{\xi\}$. It follows that there exists an orthogonal map

$$
\tilde{T}: T_{f(p)}^{\perp} M \oplus \operatorname{span}\{\xi\} \rightarrow \operatorname{span}\{\eta\} \oplus T_{g(p)}^{\perp} M
$$

such that $\tilde{T} \tilde{\beta}=\bar{\beta}$. Set

$$
\tilde{T} \xi=\eta \cos \varphi+\delta_{1} \sin \varphi,
$$

where $\delta_{1} \in \operatorname{span}\{\eta, \tilde{T} \xi\}$ satisfies $\left|\delta_{1}\right|=1$ and $\left\langle\delta_{1}, \eta\right\rangle=0$. Let $\gamma_{1} \in T_{f(p)}^{\perp} M$ be chosen so that

$$
\tilde{T} \gamma_{1}=-\eta \sin \varphi+\delta_{1} \cos \varphi
$$

We extend $\gamma_{1}$ to an orthonormal basis $\gamma_{1}, \ldots, \gamma_{d}$ of $T_{f(p)}^{\perp} M$ and define $\delta_{j}=\tilde{T} \gamma_{j}, j \geq 2$. If $\tilde{T} \xi=-\eta$, then we take $\gamma_{1}, \ldots, \gamma_{d}$ as being any orthonormal basis in $T_{f(p)}^{\perp} M$ and $\delta_{j}=\tilde{T} \gamma_{j}$. If $\tilde{T} \xi=\eta$, then the equality $\tilde{T} \tilde{\beta}=\bar{\beta}$ yields that $\left\langle\alpha^{G}, \eta\right\rangle=-\left\langle\alpha^{G}, \xi\right\rangle$ which is in contradiction with $\left\langle\alpha^{G}, \eta+\xi\right\rangle=-\langle$,$\rangle . We write$

$$
\tilde{\beta}=-\left\langle\alpha^{G}, \xi\right\rangle \xi+\left\langle\alpha^{f}, \gamma_{1}\right\rangle \gamma_{1}+\sum_{j=2}^{d}\left\langle\alpha^{f}, \gamma_{j}\right\rangle \gamma_{j}
$$

and

$$
\bar{\beta}=\left\langle\alpha^{G}, \eta\right\rangle \eta+\left\langle\left(\alpha^{G}\right)^{*}, \delta_{1}\right\rangle \delta_{1}+\sum_{j=2}^{d}\left\langle\left(\alpha^{G}\right)^{*}, \delta_{j}\right\rangle \delta_{j} .
$$


Thus, $\tilde{T} \tilde{\beta}=\bar{\beta}$ implies that

$$
\begin{gathered}
\left\langle\alpha^{G}, \eta\right\rangle=-\left\langle\alpha^{G}, \xi\right\rangle \cos \varphi-\left\langle\alpha^{f}, \gamma_{1}\right\rangle \sin \varphi \\
\left\langle\left(\alpha^{G}\right)^{*}, \delta_{1}\right\rangle=-\left\langle\alpha^{G}, \xi\right\rangle \sin \varphi+\left\langle\alpha^{f}, \gamma_{1}\right\rangle \cos \varphi \\
\left\langle\left(\alpha^{G}\right)^{*}, \delta_{j}\right\rangle=\left\langle\alpha^{f}, \gamma_{j}\right\rangle, \quad \forall j \geq 2 .
\end{gathered}
$$

From the first equation, we get

$$
\begin{aligned}
-\left\langle\alpha^{G}, \xi\right\rangle \cos \varphi-\left\langle\alpha^{f}, \gamma_{1}\right\rangle \sin \varphi & =\left\langle\alpha^{G}, \eta\right\rangle=\left\langle\alpha^{G}, \eta+\xi\right\rangle-\left\langle\alpha^{G}, \xi\right\rangle \\
& =-\langle,\rangle-\left\langle\alpha^{G}, \xi\right\rangle .
\end{aligned}
$$

Hence

Furthermore,

$$
\left\langle\alpha^{G}, \xi\right\rangle=\frac{1}{\cos \varphi-1}\left(\langle,\rangle-\left\langle\alpha^{f}, \gamma_{1}\right\rangle \sin \varphi\right) .
$$

$$
\left\langle\left(\alpha^{G}\right)^{*}, \delta_{1}\right\rangle=-\left\langle\alpha^{f}, \gamma_{1}\right\rangle+\frac{1}{1-\cos \varphi} \sin \varphi\langle,\rangle .
$$

We conclude that (1.23) holds for

$$
\begin{gathered}
\mu_{1}=-\frac{1}{1-\cos \varphi}\left(\xi+\eta \cos \varphi+\delta_{1} \sin \varphi\right), \\
\mu_{2}=\frac{-\sin \varphi}{1-\cos \varphi}(\xi+\eta)-\delta_{1} \quad \text { and } \mu_{j+1}=\delta_{j}, \quad j \geq 2 .
\end{gathered}
$$

Now let $F: M^{n} \rightarrow \mathbb{V}^{n+d+1}$ be defined by $F=J_{\zeta} \circ f$, where $\zeta \in \mathbb{V}^{n+d+1}$ is arbitrary. The second fundamental form of $F$ in $\mathbb{L}^{n+d+2}$ is given by

$$
\alpha^{F}=\alpha^{f}-\langle,\rangle \zeta
$$

As previously discussed, the proof of Theorem 1.1 will be completed once we show the following fact.

Assertion 5. There exists a smooth vector bundle isometry $\hat{T}: T_{G}^{\perp} M \rightarrow$ $T_{F}^{\perp} M$ which preserves the second fundamental forms and normal connections.

Set $\xi_{1}=\zeta, \xi_{j+1}=\gamma_{j}, 1 \leq j \leq d$, and define

$$
\hat{T}\left(\mu_{j}\right)=\xi_{j}, \quad 1 \leq j \leq d+1, \quad \hat{T}(G)=F .
$$

Clearly, $\hat{T}$ is isometric. We have that $\hat{T} \alpha^{G}(X, Y)=\alpha^{F}(X, Y)$ by (1.23) and (1.24). We claim that $\operatorname{dim} S\left(\alpha^{F}\right)=d+1$. In fact, let $L$ be the orthogonal complement of $S\left(\alpha^{F}\right)$ in $T_{F}^{\perp} M=T_{f(p)}^{\perp} M \oplus \operatorname{span}\{\zeta, F\}$. Due to the fact that the inner product in $T_{F}^{\perp} M$ is non-degenerate, it holds that $\operatorname{dim} S\left(\alpha^{F}\right)+$ $\operatorname{dim} L=\operatorname{dim} T_{F}^{\perp} M=d+2$. Take $\Gamma=\gamma+a \zeta+b F$ an arbitrary vector in $L$ with $\gamma \in T_{f(p)}^{\perp} M$. Since $\left\langle\alpha^{F}, \Gamma\right\rangle=0$ and $\langle\zeta, F\rangle=1$ we conclude that 
$\left\langle\alpha^{f}, \gamma\right\rangle-b\langle\rangle=$,0 . If $\gamma \neq 0$, then we obtain that $A_{\gamma}^{f}$ is umbilical and this contradicts the hypothesis on the conformal 1-nullity of $f$. Then $\gamma=0$ and, consequently, $b=0$. Hence $L=\operatorname{span}\{\zeta\}$ and the claim follows. By a similar argument, we also deduce that $\operatorname{dim} S\left(\alpha^{G}\right)=d+1$ due to (1.23). Thus, we have

$$
T_{G}^{\perp} M=S\left(\alpha^{G}\right) \oplus \operatorname{span}\{G\} \quad \text { and } \quad T_{F}^{\perp} M=S\left(\alpha^{F}\right) \oplus \operatorname{span}\{F\} .
$$

These facts easily imply that $\hat{T}$ is smooth. In particular, the vector field $\mu_{1}$ is smooth, because $\hat{T}\left(\mu_{1}\right)=\zeta$. It remains to be shown that $\hat{T}$ preserves the normal connections. For any vector field $\xi \in T_{G}^{\perp} M$, define $\Phi_{\xi}: T M \rightarrow T_{F}^{\perp} M$ by setting $\Phi_{\xi}(X)=\hat{T}\left(\nabla_{X}^{\perp} \xi\right)-\nabla_{X}^{\perp} \hat{T}(\xi)$. It follows easily from the Codazzi equations for $F$ and $G$ that

$$
\left\langle\alpha^{F}(Z, Y), \Phi_{\xi}(X)\right\rangle=\left\langle\alpha^{F}(Z, X), \Phi_{\xi}(Y)\right\rangle, \quad \forall X, Y, Z \in T_{p} M .
$$

In particular, for $\xi=\mu_{1}$ this yields that

$$
\left\langle\alpha^{f}(Z, Y), \Phi_{\mu_{1}}(X)\right\rangle=\left\langle\alpha^{f}(Z, X), \Phi_{\mu_{1}}(Y)\right\rangle, \quad \forall X, Y, Z \in T_{p} M,
$$

because $\left\langle\Phi_{\mu_{1}}(X), \zeta\right\rangle=\left\langle\nabla_{X}^{\frac{1}{X}} \mu_{1}, \mu_{1}\right\rangle-\left\langle\nabla_{X}^{\frac{1}{X}} \zeta, \zeta\right\rangle=0$.

We claim that $\Phi_{\mu_{1}}=0$, that is, $\mu_{1}$ is parallel in the normal connection. Suppose otherwise that $\operatorname{dim}\left(\operatorname{Im} \Phi_{\mu_{1}}\right)=r \geq 1$. In this case, we have that

$$
\left\langle\alpha^{f}(Z, X), \Phi_{\mu_{1}}(Y)\right\rangle=0, \quad \forall X \in \operatorname{ker} \Phi_{\mu_{1}}, Y, Z \in T_{p} M .
$$

Hence,

$$
\nu_{r}^{c}(p) \geq \operatorname{dim} \operatorname{ker} \Phi_{\mu_{1}}=n-r .
$$

But this is in contradiction with the hypothesis on the conformal 1-nullity of $f$ when $r=1$ and with the Proposition 1.1 when $r \geq 2$.

Now, we obtain that $\left\langle\Phi_{\xi}(X), \zeta\right\rangle=\left\langle\nabla_{X}^{\perp} \xi, \mu_{1}\right\rangle-\left\langle\nabla_{X}^{\perp} \hat{T}(\xi), \zeta\right\rangle=0$ for any vector $\xi \in T_{G}^{\perp} M$. Hence, (1.24) and (1.25) imply that

$$
\left\langle\alpha^{f}(Z, Y), \Phi_{\xi}(X)\right\rangle=\left\langle\alpha^{f}(Z, X), \Phi_{\xi}(Y)\right\rangle, \quad \forall X, Y, Z \in T_{p} M .
$$

Arguing as before we conclude that $\Phi_{\xi}=0$. According to observations made previously, Theorem 1.1 has been proved.

Corollary 1.1. Theorem 1.3 holds for $d=5$.

Proof. The form $\beta$ defined on p. 976 in $[\mathbf{C}-D$ ] always satisfies $\beta(Z, Z) \neq 0$ for all $0 \neq Z \in T M$. Given $X \in R E(\beta)$, since $n>2 d+2$, there exists $Z \neq 0$ such that $Z \in \operatorname{ker} \beta(X)$. Since $\beta$ is flat, $\beta(Z, Y) \in U(X)$ for all $Y$ and $U(X)$ is isotropic, we deduce that $\beta(Z, Z) \in S(\beta) \cap S(\beta)^{\perp}$. Thus $\beta$ admits a decomposition as in the Main Lemma 2.2, p. 967. The inequality on the dimension of $N\left(\beta_{2}\right)$ follows from Sublemma 1.2 in this paper because $\operatorname{dim} W_{2} \leq 10$ for $d=5$. The remainder of the proof is identical. 
Remark 1.1. In Theorem 1.1, the hypothesis $\tau_{f}^{c}(p) \geq 3$ implies that $n \geq$ $3 d$. For $d=1$ and $n<2 d+3$, we have that either $n=3$ or $n=4$. An immersion of $M^{3}$ into $\mathbb{R}^{4}$ always satisfies $\nu_{1}^{c} \geq 1$. Cartan $([\mathbf{C a} 2])$ gave examples of immersions $M^{4}$ into $\mathbb{R}^{5}$ which have four distinct principal curvatures at each point, hence $\tau_{f}^{c}=4$ and $\nu_{1}^{c}=1$, which are not conformally rigid. For $d=2$ and $n<2 d+3$, we have that $n=6$ and it is not known whether an immersion of $M^{6}$ into $\mathbb{R}^{8}$ with $\tau_{f}^{c}=3, \nu_{1}^{c} \leq 3$, and $\nu_{2}^{c} \leq 1$ is conformally rigid.

Remark 1.2. The beginning of the argument to prove that the form $\beta_{2}$ is zero in Assertion 3.3 of [C-D] says that there exist orthonormal bases of $T_{f(p)}^{\perp} M$ and $T_{g(p)}^{\perp} M$ with some special properties which, in fact, may not be satisfied under the conditions there. The argument in Assertion 3 to prove that the form $\omega_{2}$ is zero corrects the one in $[\mathbf{C}-\mathbf{D}]$.

To finish this section, we point out that in Theorem 1.1 the requirement on $\nu_{s}^{c}(p)$ can not be dropped. First, we prove that any product of spheres is conformally deformable. Fix positive integers $d \geq 2$ and $k_{i}, 1 \leq i \leq$ $d$, and arbitrary positive real numbers $\lambda_{i}, 1 \leq i \leq \bar{d}$. Denote by $\mathbb{S}_{\lambda_{i}}^{k_{i}}$ the $k_{i}$-dimensional sphere centered in the origin and radius $\lambda_{i}$. Let $M^{n}, n=$ $\sum_{i=1}^{d} k_{i}$, be the riemannian product with factors $\mathbb{S}_{\lambda_{i}}^{k_{i}}, 1 \leq i \leq d$. Define the product immersion $f: M^{n} \rightarrow \mathbb{R}^{n+d}$ by setting $f=I_{1} \times I_{2} \times \cdots \times I_{d}$ where $I_{i}: \mathbb{S}_{\lambda_{i}}^{k_{i}} \rightarrow \mathbb{R}^{k_{i}+1}$ is the inclusion. We have that $f(M) \subset \mathbb{S}_{\lambda}^{n+d-1}$ where $\lambda=\sqrt{\sum_{i=1}^{d} \lambda_{i}^{2}}$. The immersion $f$ is conformally deformable, that is, it is not conformally rigid. In fact, the induced metric on $M$ by $f$ is the same one induced by $\mathbb{S}_{\lambda}^{n+d-1}$, namely, the natural product metric. Identify $\mathbb{R}^{n+d-1}$ with the hyperplane of $\mathbb{R}^{n+d}$ whose points $x=\left(x_{1}, x_{2}, \ldots, x_{n+d}\right)$ satisfy $x_{n+d}=0$. Let $\Pi: \mathbb{S}_{\lambda}^{n+d-1}-\left\{p_{\lambda}\right\} \rightarrow \mathbb{R}^{n+d-1}$ be the stereographic projection raised from the point $p_{\lambda}=(0, \ldots, 0, \lambda)$ which is given by

$$
\Pi(p)=p_{\lambda}+\frac{\lambda}{\lambda-p_{n+d}}\left(p-p_{\lambda}\right) \text { where } p=\left(p_{1}, p_{2}, \ldots, p_{n+d}\right) .
$$

The map $\Pi$ is a conformal diffeomorphism with conformal factor $\frac{\lambda}{\lambda-p_{n+d}}$, that is,

$$
\left|\left(\Pi_{*}\right)_{p} v\right|^{2}=\frac{\lambda^{2}}{\left(\lambda-p_{n+d}\right)^{2}}|v|^{2}
$$

for any $p \in \mathbb{S}_{\lambda}^{n+d-1}-\left\{p_{\lambda}\right\}$ and $v \in T_{p}\left(\mathbb{S}_{\lambda}^{n+d-1}-\left\{p_{\lambda}\right\}\right)$. Observe also that $p_{\lambda} \notin M^{n}$ since $d \geq 2$. Let $\varphi: M^{n} \rightarrow \mathbb{R}^{n+d-1} \subset \mathbb{R}^{n+d}$ be the restriction of $\Pi$ to $M^{n}$. Consider also the isometric immersion $\phi: \mathbb{R}^{n+d-1} \rightarrow$ $\mathbb{S}_{1}^{1} \times \mathbb{R}^{n+d-2} \subset \mathbb{R}^{n+d}$ defined by

$$
\phi\left(x_{1}, x_{2}, \ldots, x_{n+d-1}, 0\right)=\left(\cos x_{1}, \sin x_{1}, x_{2}, \ldots, x_{n+d-1}\right) .
$$


Define $g: M^{n} \rightarrow \mathbb{R}^{n+d}$ as $g=\phi \circ \varphi$. It follows easily that $f$ and $g$ are conformal. Recall that the inversion with respect to the unit sphere centered at $p_{o}$ is the conformal transformation $I_{p_{o}}(q)=p_{o}+\left[\left(q-p_{o}\right) /\left|q-p_{o}\right|^{2}\right]$, $q \in \mathbb{R}^{n+d}-\left\{p_{o}\right\}$, an isometry of $\mathbb{R}^{n+d}$ is a map $\Im$ such that $\Im(q)=O(q)+w$, where $O$ is an orthogonal map of $\mathbb{R}^{n+d}$ and $w$ is a fixed vector in $\mathbb{R}^{n+d}$, and a dilatation $D_{\alpha}$ is a transformation of $\mathbb{R}^{n+d}$ such that $D_{\alpha}(q)=\alpha q$ for some positive real constant $\alpha$. Recall further that by Liouville's theorem (see $[\mathbf{d C}]$ ) every conformal diffeomorphism $\Upsilon$ from an open subset $U$ of $\mathbb{R}^{n+d}$ to an open subset $V$ of $\mathbb{R}^{n+d}$ is the restriction to $U$ of a composition of inversions, dilatations and isometries, at most one of each. We claim that there is not a such conformal diffeomorphism $\Upsilon$ satisfying $g=\Upsilon \circ f$. In fact, it is not difficult to see that there exist one inversion $I_{p_{o}}$, one dilatation $D_{\alpha}$ and one isometry $\Im$ such that $\Upsilon$ is the restriction to $U$ either of the composition $\Im \circ D_{\alpha} \circ I_{p_{o}}$ or the composition $\Im \circ D_{\alpha}$. Now suppose on the contrary that $g=\Upsilon \circ f$ for some conformal diffeomorphism $\Upsilon$. We have to analyze two cases.

Case i). The conformal map $\Upsilon$ is the restriction to $U$ of the composition $\Im \circ D_{\alpha} \circ I_{p_{o}}$. In this case, extending $\Upsilon$ if necessary we can assume that $U=\mathbb{R}^{n+d}-\left\{p_{o}\right\}$. The conformal factor $\beta$ of $\Upsilon$ is $\beta(q)=\alpha /\left|q-p_{o}\right|^{2}$. Since $g=\Upsilon \circ f$ on $M^{n}$ we have that

$$
\left(\phi_{*}\right)_{\varphi(p)}\left(\varphi_{*}\right)_{p} v=\left(g_{*}\right)_{p} v=\left(\Upsilon_{*}\right)_{p} v
$$

and, consequently,

$$
\frac{\lambda^{2}}{\left(\lambda-p_{n+d}\right)^{2}}|v|^{2}=\left|\left(g_{*}\right)_{p} v\right|^{2}=\left|\left(\Upsilon_{*}\right)_{p} v\right|^{2}=\beta(p)^{2}|v|^{2}
$$

for all $v \in T_{p} M$. This yields that

$$
\frac{\lambda}{\lambda-p_{n+d}}=\beta(p)=\frac{\alpha}{\left|p-p_{o}\right|^{2}}
$$

for all $p \in M^{n}$. Therefore,

$$
\lambda\left|\gamma(t)-p_{o}\right|^{2}=\alpha\left(\lambda-\gamma_{n+d}(t)\right)
$$

for any curve $\gamma(t)$ in $M^{n}$ with $\gamma(t)=\left(\gamma_{1}(t), \ldots, \gamma_{n+d}(t)\right)$. Taking derivatives in the last equality, we have that

$$
2 \lambda\left\langle\gamma^{\prime}(t), \gamma(t)-p_{o}\right\rangle=-\alpha \gamma_{n+d}^{\prime}(t) .
$$

The vector $\gamma(t)$ is orthogonal to $\gamma^{\prime}(t)$ since $\gamma(t) \in \mathbb{S}_{\lambda}^{n+d-1}$ for all $t$. Then, we obtain that

$$
\left\langle\gamma^{\prime}(t), 2 \lambda p_{o}-\alpha e_{n+d}\right\rangle=0
$$

from (1.27) where $e_{n+d}=(0, \ldots, 0,1)$. So the vector $\gamma^{\prime}(t)$ belongs to the hyperplane through the origin and orthogonal to the vector $2 \lambda p_{o}-\alpha e_{n+d}$, for any curve $\gamma(t)$ in $M^{n}$. Since the vectors tangent to $M^{n}$ span $\mathbb{R}^{n+d}$ we 
have that $2 \lambda p_{o}-\alpha e_{n+d}=0$. Thus, $p_{o i}=0,1 \leq i \leq n+d-1$, and $\alpha=2 \lambda p_{o(n+d)}$. At a point such that $p_{n+d}=0$ we deduce that $\alpha=2 \lambda^{2}$ from (1.26). Consequently, $p_{o}=p_{\lambda}$. Hence,

$$
\Upsilon(q)=\left(\Im \circ D_{\alpha} \circ I_{p_{o}}\right)(q)=2 \lambda^{2} O\left(p_{\lambda}\right)+\frac{2 \lambda^{2}}{\left|q-p_{\lambda}\right|^{2}} O\left(q-p_{\lambda}\right)+w
$$

for all $q \in \mathbb{R}^{n+d}-\left\{p_{\lambda}\right\}$. The equality $\Upsilon(p)-\Upsilon(-p)=g(p)-g(-p)$ on $M^{n}$ implies that

$$
2 O(p)=\phi(p)-\phi(-p)
$$

at a point such that $p_{n+d}=0$ since the restriction to $M^{n} \cap \mathbb{R}^{n+d-1}$ of $\varphi$ is the identity. Taking length in the last equality, we conclude that $\sin ^{2} p_{1}=p_{1}^{2}$ if $p=\left(p_{1}, \ldots, p_{n+d-1}, 0\right)$. Choosing a point such that $p_{1} \neq 0$ we have obtained a contradiction.

Case ii). The conformal map $\Upsilon$ is the restriction to $U$ of $\Im \circ D_{\alpha}$. In this case, we obtain that $\lambda /\left(\lambda-p_{n+d}\right)=\alpha$ for all $p \in M^{n}$ by (1.26). Consequently, $p_{n+d}$ is constant through $M^{n}$. But this is a contradiction.

Now we compute $\nu_{s}^{c}$ for $f=I \times I \times \cdots \times I$, where $I: \mathbb{S}_{1}^{r} \rightarrow \mathbb{R}^{r+1}$ is the inclusion. In this case, $M=\mathbb{S}_{1}^{r} \times \mathbb{S}_{1}^{r} \times \cdots \times \mathbb{S}_{1}^{r}$ and $M=f(M) \subset \mathbb{S}_{\sqrt{d}}^{n+d-1}$ with $n=r d$. Given $p=\left(p_{1}, p_{2}, \ldots, p_{d}\right) \in M^{n}$ and $s, 1 \leq s \leq d$, consider at $T_{f(p)}^{\perp} M$ the points

$$
q_{1}=p, q_{i}=\left(0, \ldots, 0, p_{i}, p_{i+1}, \ldots, p_{d}\right), 2 \leq i \leq s,
$$

and $U^{s}$ defined as $U^{s}=\operatorname{span}\left\{q_{1}, \ldots, q_{s}\right\}$. It is not difficult to see that there exists $\eta \in U^{s}$ such that $\operatorname{dim} N\left(\alpha_{U^{s}}^{f}-\langle,\rangle \eta\right)=n-r(s-1)$. Fix an orthonormal basis $E_{i}^{k}, 1 \leq i \leq r$, on the tangent space of $\mathbb{S}_{1}^{r}$ at $p$ for each $k, 1 \leq k \leq d$. The vectors $E_{i}=\left(E_{i}^{1}, E_{i}^{2}, \ldots, E_{i}^{d}\right), 1 \leq i \leq r$, in $T_{f(p)}^{\perp} M$ are linearly independent. The normal space $T_{f(p)}^{\perp} M$ is spanned by $p_{k}, 1 \leq k \leq d$. The second fundamental form of $f$ satisfies $A_{p_{k}} E_{i}=$ $\left(0, \ldots, 0,-E_{i}^{k}, 0, \ldots, 0\right), 1 \leq i \leq r, 1 \leq k \leq d$. This show that $\tau_{f}^{c}(p)=r$. Consequently, we conclude that $\nu_{s}^{c}(p)=n-(s-1) r$ by Proposition 1.1. Observe that always $\nu_{1}^{c}=n$. If we take $r=3$ and $d \geq 3$, we obtain that $\nu_{s}^{c}(p)>n-2 s-1,1 \leq s \leq 3$. This fact shows that the assumption on $\nu_{s}^{c}(p)$ is necessary.

\section{Proof of Theorem 1.2.}

First, we recall from $[\mathbf{C}-\mathbf{D}]$ the following concept.

Definition 2.1. Given $s, 1 \leq s \leq d$, the $s$-nullity of an isometric immersion $f: M^{n} \rightarrow \tilde{M}^{n+d}$ at $p \in M^{n}$ is the integer

$$
\nu_{s}(p)=\max _{U^{s} \subseteq T_{f(p)}^{\perp} M}\left\{\operatorname{dim} N\left(\alpha_{U^{s}}^{f}\right)\right\} .
$$


Lemma 2.1. If $\tau_{f}^{k}(p) \geq r$, then the dimension of the tangent subspace $L(U)$ defined as $L(U)=\operatorname{span}\left\{A_{\mu} X: \mu \in U^{\ell}, X \in T_{p} M\right\}$ is at least $(k+\ell-d) r$ for any $\ell$-dimensional subspace $U^{\ell} \subset T_{f(p)}^{\perp} M, \ell \geq 1$.

Proof. Take $\xi_{1}, \ldots, \xi_{k} \in T_{f(p)}^{\perp} M$ and let $X_{1}, \ldots, X_{r} \in T_{p} M$ be such that the vectors $A_{\xi_{i}} X_{j}, 1 \leq i \leq k, 1 \leq j \leq r$, are linearly independent. Recall that the vectors $\xi_{1}, \ldots, \xi_{k}, X_{1}, \ldots, X_{r}$ are linearly independent.

We claim that the vectors $A_{\gamma_{i}} X_{j}, 1 \leq i \leq k^{\prime}, 1 \leq j \leq r$, are linearly independent when we take $\gamma_{1}, \ldots, \gamma_{k^{\prime}} \in \operatorname{span}\left\{\xi_{1}, \ldots, \xi_{k}\right\}, k^{\prime} \leq k$, linearly independent. In fact, consider a basis $\gamma_{1}, \ldots, \gamma_{k}$ of $\operatorname{span}\left\{\xi_{1}, \ldots, \xi_{k}\right\}$ which extends $\gamma_{1}, \ldots, \gamma_{k^{\prime}}$. Define the $k \times k$-matrix $B=\left(b_{i j}\right)$ by setting $\xi_{i}=$ $\sum_{h=1}^{k} b_{h i} \gamma_{h}$. It is not difficult to see that

$$
\sum_{i, j=1}^{k, r} a_{i j} A_{\xi_{i}} X_{j}=\sum_{i, j=1}^{k, r} c_{i j} A_{\gamma_{i}} X_{j}
$$

for arbitrary real numbers $a_{i j}, 1 \leq i \leq k, 1 \leq j \leq r$, being $C=\left(c_{i j}\right)$ given by $C=B A$ with $A=\left(a_{i j}\right)$. Thus, the vectors $A_{\xi_{i}} X_{j}$ and $A_{\gamma_{i}} X_{j}$ span the same subspace and the claim follows.

We can assume that $(k+\ell-d) \geq 1$. Otherwise, Lemma 2.1 is immediate. The subspace $\bar{L}=U \cap \operatorname{span}\left\{\xi_{1}, \ldots, \xi_{k}\right\}$ satisfies $\operatorname{dim} \bar{L} \geq(k+\ell-d)$ by (1.13). If $\gamma_{1}, \ldots, \gamma_{(k+\ell-d)}$ are vectors linearly independent in $\bar{L}$, then the $(k+\ell-d) r$ vectors $A_{\gamma_{i}} X_{j} \in L(U), 1 \leq i \leq(k+\ell-d), 1 \leq j \leq r$, are linearly independent from the claim. Hence, Lemma 2.1 has been proved.

Proposition 2.1. Let $f: M^{n} \rightarrow \tilde{M}^{n+d}$ be an isometric immersion. If $\tau_{f}^{k}(p) \geq r$, then $\nu_{s}(p) \leq n-(k+s-d) r$ for $1 \leq s \leq d$.

Proof. If $k+s-d \leq 0$ then the conclusion is immediate. Assume that $k+s-d \geq 1$. Let $U^{s} \subseteq T_{f(p)}^{\perp} M$ be such that $\nu_{s}(p)=\operatorname{dim} N\left(\alpha_{U^{s}}^{f}\right)$. We have that $\left\langle A_{\mu} X, v\right\rangle=0$ for all $\mu \in U^{s}, v \in N\left(\alpha_{U^{s}}^{f}\right)$ and $X \in T_{p} M$, that is, $L(U) \subseteq N\left(\alpha_{U^{s}}^{f}\right)^{\perp}$. Therefore, using Lemma 2.1, we deduce that

$$
n-\nu_{s}(p)=\operatorname{dim} N\left(\alpha_{U^{s}}^{f}\right)^{\perp} \geq \operatorname{dim} L(U) \geq(k+s-d) r,
$$

and Proposition 2.1 follows.

Proof of Theorem 1.2. We only have to deal with the case of codimension $d \geq 6$ since the proof follows from a result in [C-D] for $d \leq 5$. If $\tau_{f}^{d-1}(p) \geq 3$, then $n \geq 3 d-3$. We can assume that $n \geq 2 d+1$. Otherwise, we obtain that $2 \leq d \leq 3$. For $d=2$ and $d=3$, we conclude that $n=3,4$ and $n=6$, respectively. But both cases are not possible because we are assuming 
$\nu_{2} \leq n-5$ and $\nu_{3} \leq n-7$. It follows from Proposition 2.1 for $k=d-1$ that $\nu_{s}(p) \leq n-2 s-1$ when $s \geq 4$. Hence, their result applies for $d \leq 5$.

Let $g: M^{n} \rightarrow \mathbb{R}^{n+d}$ be another immersion isometric to $f$. Given $p \in M$, let

$$
W=T_{f(p)}^{\perp} M \oplus T_{g(p)}^{\perp} M
$$

be endowed with the natural metric of type $(d, d)$ who is negative definite in $T_{f(p)}^{\perp} M$, and define the symmetric bilinear form $\beta: T M \times T M \rightarrow W$ by

$$
\beta=\alpha^{f}+\alpha^{g} .
$$

The Gauss equations for $f$ and $g$ imply that $\beta$ is flat. Since $\tau_{f}^{d-1}(p) \geq 3$, we can fix vectors $\xi_{1}, \ldots, \xi_{d-1}$ of $T_{f(p)}^{\perp} M$ and $X_{1}, X_{2}, X_{3} \in V$ so that the vectors $A_{\xi_{i}} X_{j}, 1 \leq i \leq d-1,1 \leq j \leq 3$, are linearly independent. To see that $\beta$ is null we proceed exactly as in Lemma 1.1 with the $(n-3 d+3)$ dimensional subspace

$$
\tilde{L}=\left(\operatorname{span}\left\{A_{\xi_{i}} X_{j}, 1 \leq i \leq d-1,1 \leq j \leq 3\right\}\right)^{\perp},
$$

with $d$ instead of $d+1$ and Proposition 2.1 instead of Proposition 1.1.

The remainder of the proof is part of the argument for Theorem 1.4 in [C-D].

Remark 2.1. The hypothesis in Theorem 1.2, as mentioned in the introduction, are less restrictive than those in Allendoerfer's theorem. In fact, the assumption $\tau_{f}^{d}(p) \geq 3$ implies that $\tau_{f}^{d-1}(p) \geq 3$ and that $\nu_{s}(p) \leq n-3 s$ for $1 \leq s \leq d$; c.f. Proposition 4.6 in [Da].

\section{References}

[Al] Carl B. Allendoerfer, Rigidity for spaces of class greater than one, Amer. J. of Math., 61 (1939), 633-644.

[Ca1] E. Cartan, La déformation des hypersurfaces dans l'espace conforme réel a $n \geq 5$ dimensions, Bull. Soc. Math. France, 45 (1917), 57-121.

[Ca2] - Sur certaines hypersurfaces de l'espace conforme réel a cinq dimensions, Bull. Soc. Math. France, 46 (1918), 84-105.

[Da] M. Dajczer, Submanifolds and Isometric Immersions, Math. Lec. Series, 13, Publish or Perish, Inc. Houston, 1990.

[D-T] M. Dajczer and R. Tojeiro, A rigidity theorem for conformal immersions, Indiana Univ. Math. J., 46 (1997), 491-504.

[dC] M. do Carmo, Riemannian Geometry, Birkhäuser Boston, 1992. 
[C-D] M. do Carmo and M. Dajczer, Conformal rigidity, Amer. J. of Math., 109 (1987), 963-985.

Received August 24, 1999.

Universidade Estadual do Rio DE JANEIRO-UERJ

Departamento de Estruturas Matemáticas-IME

20550-013, Rio DE JANEIRO

BRAZIL

E-mail address: sergiol@ime.uerj.br 